\title{
A Study of Sustainability Adoption Trends in the Transportation Market
}

\author{
Marta Jaen Ventura, Christine Toh, Carlos Parra, and Gul Kremer \\ Industrial Engineering and Manufacturing Department, \\ Pennsylvania State University \\ 16801 University Park, PA \\ $\{m x v 176, \cot 5015$, cjp246, gek3 \} @psu.edu
}

\begin{abstract}
Due to an increasing concern for sustainability, automobile manufacturers have been introducing hybrid variants of popular vehicles in an attempt to reduce the environmental impact of vehicles. However, it is still unclear how this 'hybridization' of popular car models affects the overall success of the vehicle. This paper seeks to address these gaps in knowledge by implementing a demand modeling approach, game theory framework, and a technology forecasting model. In the demand model, it was found that the percent of hybrid cars sold, the year and the model of the car are significant predictors of the total sales. In the game theory model, all possible scenarios suggest that an increase in hybrid sales would encourage market share growth. Lastly, using the technology forecasting model, S-curves were generated to show how the energy impact score (EIS) of automobile engines decreases over time as technology improves, and provide justification for the investment in hybrid engine development.
\end{abstract}

\section{$1 \quad$ Introduction}

Although the hybrid electric vehicle was first developed as an alternative to traditional internal combustion engine vehicles in as early as the 1900s, it has only recently gained momentum as a core segment of the automobile industry [1]. Hybrid Electric Vehicles (HEVs) combine the conventional internal combustion engine and an electric propulsion system that that uses energy stored in a battery. This combination allows the vehicle to have same power and range as other vehicles while getting better fuel economy and lowering negative emissions since the combustion engine is reduced in size and capacity [2]. Although only comprising of a small percentage of the automobiles that are driven today, the United States is home to the largest fleet of HEVs [3], making it a significant part of any sustainability adoption consideration.

Since the HEVs are considered to be a step in the direction for a more sustainable driving future [4], an increasing number of consumers are adopting hybrid technology. Thus, automobile manufacturers are beginning to offer hybrid variations of their top selling models. Examples of this 'hybridization' of popular vehicle models include the Toyota Camry, Honda Accord, and Nissan Altima. While this strategy provides consumers with a more sustainable option for trusted vehicle 
models, it is still unclear how this 'hybridization' affects consumer adoption of HEVs. In other words, since automobile makers largely influence consumer behavior (i.e., availability can drive desirability), the increase in hybrid sales should be investigated for its impact on the automobile maker's total sales. In addition, consumer perception of a particular automobile maker's brand could be influenced by this 'hybridization', ultimately affecting the success of the vehicle. Thus, this paper seeks to examine the role of HEV adoption on the success of a model of vehicle by using a Demand Modeling framework, as has been previously done by other researchers who seek to facilitate product planning in competitive markets $[5,6]$.

In addition to the proportion of hybrid cars sold by a single automobile maker, the behavior of other automobile makers have the potential to influence overall sales. One method of analyzing the market dynamics of a few major players is through the Game Theory framework. Formally, game theory involves the study of "mathematical models of conflict and cooperation between intelligent rational decision-makers" [7]. The rational decision makers to be considered in this paper are the individual automobile manufacturers, and game theory can be used to uncover the most effective and profitable strategy to be taken by multiple players (automobile manufacturers). Thus, one of the main goals of this paper is to examine the relative payoffs (sales) for each major automobile manufacturer in when considering varying levels of hybrid car sales.

While the impact of hybrid sales and competitors' behavior can certainly affect the success of a particular vehicle, the life-cycle of hybrid technology needs to also be examined. This is important for justifying investment in HEVs, given that most all technologies are surpassed by new technologies eventually. One method of analyzing the life-cycle of technologies is through the use of S-curves that depict how the technology has evolved over time [8]. The start of the S-curve depicts "market adoption" that relates to the birth of a new market opportunity. In this specific case, Toyota Camry first introduced hybrid cars in 2006. Typically, "early adopters" are consumers that are interested in testing and trying out new products. Following this phase, the technology moves into a mass manufacturing and distribution phase. Lastly, as the market matures, at which point "late adopters" who are risk adverse, begin to purchase the product. Therefore, this paper seeks to examine the growth of hybrid technology over time and provides justification for investing in a larger proportion of hybrid sales within the next ten years.

The main objective of this paper is to explore trends in hybrid technology adoption and examine the impact of competitors' behavior on the success of different models of vehicles. Therefore, this paper seeks to address the following research questions: (1) How does the percentage of hybrid cars affect the overall sales for that model? (2) How do competitors' behavior with regards to the proportion of hybrid cars being sold affect overall sales? (3) What is the future of hybrid technology and is there justification for continual investment in HEVs?

\section{$2 \quad$ Methodology}

In order to answer our research questions, a method of analyzing the current trends in hybrid vehicle technology and predicting the competitive landscape is presented in this 
section. Since the current problem involves uncovering trends in hybrid technology, three different example vehicles are used to illustrate the process, namely, the Toyota Camry, Honda Accord, and Nissan Altima vehicles. These vehicles were chosen because of the similarities in quality (belonging to the same 'tier' within each brand), and the availability of sales data. In addition, all three models are available as nonhybrid and hybrid alternatives. Thus, the first step of the proposed methodology involves utilizing a Demand Modeling approach to analyze the relationship between the proportion of hybrid cars sold and the total sales. Next, a Game Theory framework is implemented in order to gain a better understanding of the impact of competitors' behavior on sales. Lastly, the future of hybrid technology is analyzed from a sustainability perspective using Technology Forecasting. These steps are demonstrated and discussed in the following sections.

\subsection{Demand Modeling of Hybrid Sales}

The first step in determining the best strategy for maximizing sales is to examine the impact of various factors on the total sales of the model of vehicle. In particular, the proportion of hybrid sales is studied in this paper since it provides a normalized indication of the amount of hybrid cars being purchased for each model. In other words, we seek to determine if the proportion of hybrid sales can significantly predict total sales for the three chosen models of vehicles. Thus, the amount of sales of the hybrid variations of each model of vehicle $\left(\mathrm{S}_{\text {hybrid }}\right)$, as well as the total sales of each model of vehicle $\left(S_{\text {total }}\right)$ were obtained through the companies' sales reports. Then, the proportion of hybrid sales $\left(\mathrm{P}_{\text {hybrid }}\right)$ is simply computed using Equation 1 for the years that each model had a hybrid alternative available.

$$
P_{\text {hybrid }}=\frac{S_{\text {hybrid }}}{S_{\text {total }}}
$$

A linear regression analysis was then performed using $S_{\text {total }}$ as the dependent variable, and $\mathrm{P}_{\text {hybrid, }}$ model, and year as independent variables. The year was included as a variable in the analysis in order to account for fluctuations in consumer behavior throughout the years. Furthermore, since the hybrid variants of the different car models were introduced at different points in time (different year ranges), the years that saw both regular gas-powered engines and hybrid variants of the same model were included in the model. For example, from the year 2007 to 2010, Toyota produced both regular gas-powered Toyota Camrys and Hybrid Toyota Camrys. Thus, the year ranges were adjusted for each car model accordingly. In addition, the model variable was included in the analysis in order to account for inherent differences in sales between different vehicle models. Since the model variable is categorical in nature (Toyota Camry, Honda Accord, and Nissan Altima), 'Dummy Coding' was used to analyze the variable in linear regression. The total sales for each vehicle model, the model's hybrid sales, and the proportion of hybrid sales for each model are shown in Table 1. 
Table 1. Proportion of hybrid sales computed using sales of hybrid vehicles and total sales for each model of vehicle, for each year

\begin{tabular}{|l|l|l|l|l|}
\hline Vehicle Model & Year & $\mathbf{S}_{\text {hvbrid }}$ & $\mathbf{S}_{\text {total }}$ & $\mathbf{P}_{\text {hvbrid }}$ \\
\hline Toyota Camry & 2007 & 24477 & 418631 & 0.1151 \\
\cline { 2 - 5 } & 2008 & 6272 & 430345 & 0.0144 \\
\cline { 2 - 5 } & 2009 & 22887 & 333937 & 0.0641 \\
\cline { 2 - 5 } & 2010 & 14587 & 313217 & 0.0445 \\
\hline \multirow{3}{*}{ Honda Accord } & 2004 & 1061 & 358709 & 0.00274 \\
\cline { 2 - 5 } & 2005 & 16826 & 352467 & 0.0456 \\
\cline { 2 - 5 } & 2006 & 5598 & 348843 & 0.0158 \\
\hline Nissan Altima & 2007 & 8388 & 276374 & 0.0295 \\
\cline { 2 - 5 } & 2008 & 8819 & 260849 & 0.0327 \\
\cline { 2 - 5 } & 2009 & 9357 & 194211 & 0.0460 \\
\cline { 2 - 5 } & 2010 & 6710 & 22253 & 0.0293 \\
\hline
\end{tabular}

The results of the linear regression analysis conducted to model the impact of the proportion of hybrid sales revealed a significant relationship between total sales and the proportion of hybrid sales, model, and year variables $\left(\mathrm{R}^{2}=0.90, \mathrm{p}<0.00\right)$. This result indicates that the proportion of hybrid sales indeed affects the total sales of the vehicle. In addition, differences in the model and year of the vehicle can be said to affect the total sales of the vehicle as well. This is unsurprising since different vehicle models are bound to have different sales figures, affected by changes in consumer behavior from year to year. In particular, total sales appear to decrease following each year, but tend to increase as the proportion of hybrid sales increase, see Equation 2.

$$
S_{\text {total }}=42566452-20970(\text { Year })+323273\left(S_{\text {hybrid }}\right)-71814.87(\text { Model })
$$

\subsection{Implementation of Game Theory Framework}

Implementing game theory is a good way to analyze how the sales of a car will be impacted depending on which "move" or policy the competitors make. From demand modeling, it was found that overall sales were influenced by the percentage of hybrid cars the company produced. Taking this information into account, a game theory scenario was constructed where two companies were evaluated at two different levels of percentage of hybrid cars produced, out of their total number of cars. The two levels chosen were $3 \%$ and $10 \%$. There were three pairwise comparisons performedone for each pair of vehicles (Toyota Camry, Honda Accord, Nissan Altima). The assumed strategy of the game was to maximize market share among the competing companies, by examining all perceivable strategies and their corresponding payoffs for each manufacturer [9]. The values were obtained directly from the multiple-linear regression developed for demand modeling. A unique Nash Equilibrium point (NE) was found, which means that neither company has an incentive to deviate from this strategy, thus both companies should be at $10 \%$ hybrid in order to increase their market shares, regardless of the strategy of the opponent. This result was found when performing pairwise comparisons of all car companies, see Figure 1. This is an 
indication that hybrid technology is becoming a vital segment of the automobile industry, and could likely benefit automobile makers even in a competitive environment. It should be noted that Toyota Camry has the highest market share values regardless of the 'game scenario', and can be said to 'dominate' the market, possibly due to the overall brand size and popularity.
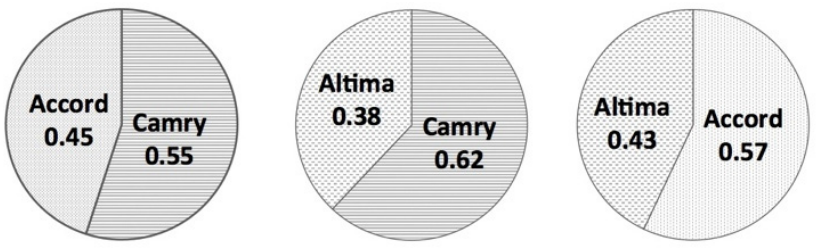

Fig. 1. Market share values for the NE point across all pairwise comparison scenarios

\subsection{Technology Forecasting of Hybrid Engines}

In order to analyze the technology life-cycle of hybrid engines, the Energy Impact Score (EIS) was used to measure the sustainability of the engine. This metric is used as an industry standard for measuring the number of barrels of petroleum consumed by a vehicle in one year [10]. Thus, the lower the EIS, the more sustainable the vehicle is said to be. The 'growth' of the technology, or in this case, the decrease in the EIS, provides an indication of trends in the sustainability of vehicle engines, and can serve as a method of predicting the sustainability of these engines. This trend is captured using S-curves, a mathematical function that models changes in parameters over time using a logistic form. In our study, the EIS generates a backwards S-curve, because the EIS decreases over time, as technology improves. Therefore, the logistic growth functions used in this paper are monotonically decreasing, reflecting and improvement in the EIS score, as seen in Equation 3.

$$
\frac{F}{F-P}=e^{\propto t+\beta}
$$

Where $\mathrm{F}$ is the Energy Impact Score, $\mathrm{P}$ is the saturation point (determined through optimization), and $\alpha$ and $\beta$ are coefficients of the exponential function. Then, for every point in the data set, we compute $\frac{F}{F-P}$ as well as $\ln \left(\frac{F}{F-P}\right)$. This transformed form of the exponential function is shown in Equation 4.

$$
\ln \left(\frac{F}{F-P}\right)=\propto t+\beta
$$

Values of $\ln \left(\frac{F}{F-P}\right)$ are plotted to find the slope, $\propto$, and intercept, $\beta$. Equation 5 is then solved for $\mathrm{F}$, treating this new value as an estimator, $\hat{F}$.

$$
\widehat{F}=\frac{-\left(P * e^{\alpha t+\beta}\right)}{\left(1-e^{\alpha t+\beta}\right)}
$$


The points for $\hat{F}$ are plotted to visually illustrate the trend of this technology and how it evolves, if it continues at its current pace. The sum of square error between the actual data point (EIS) and $\hat{F}$ is then minimized to find the lowest energy impact score that a car can achieve. The S-curves is created for the hybrid car engines as well as the non-hybrid car engines and are shown in Figures 2 and 3. Based on the S-curve, the lowest EIS that hybrid cars can achieve is 6.68, around year 2065. Effectively, hybrid cars achieve $95 \%$ of their potential for sustainability around the year 2025 (EIS = 7.01). For non-hybrid cars, the lowest EIS that can be achieved is 11.86, which saturates around year 2053. Non-hybrid cars achieve $95 \%$ of this value around the year $2016($ EIS $=12.45)$.

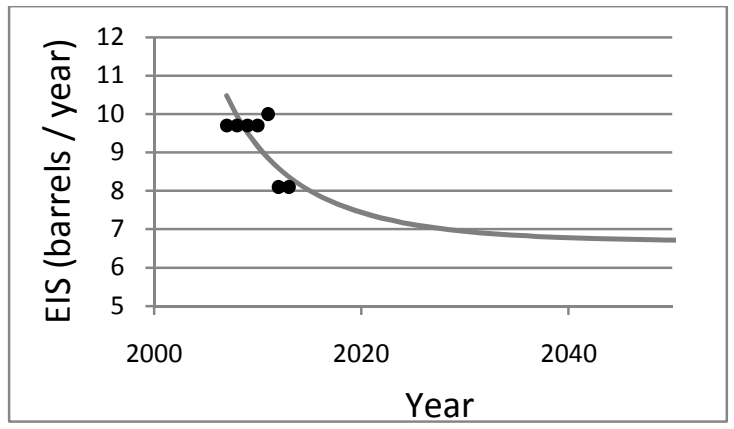

Fig. 2. S-curve of EIS for hybrid vehicles

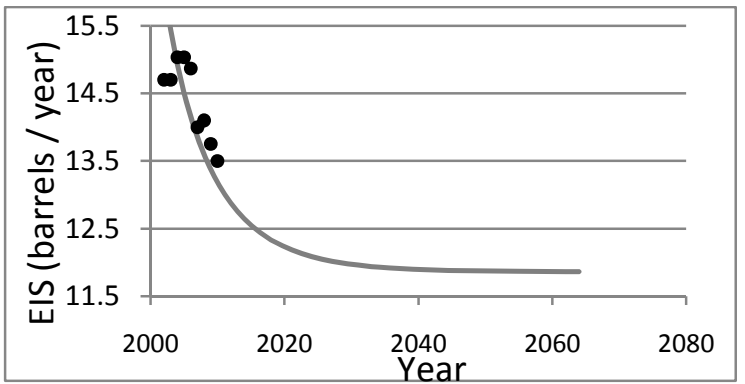

Fig. 3. S-curve for EIS for non-hybrid vehicles

To create an S-curve that combined the S-curves of hybrid and non-hybrid into one growth by means of weighted averages, a new EIS metric needed to be formulated. For the years where the hybrid and non-hybrid cars overlap (2007-2012), we compute a weighted EIS value (WEIS) for each year i, see Equation 6.

$$
W E I S_{i}=E I S_{\text {non-hybrid }}\left(S_{\text {non-hybrid }}\right)+\operatorname{EI} S_{\text {hybrid }}\left(S_{\text {hybrid }}\right)
$$

Where EIS $S_{\text {non-hybrid }}$ and EIS $S_{\text {hybrid }}$ correspond to the EIS values of the respective types of vehicles, and $S_{\text {non-hybrid }}$ and $S_{\text {hybrid }}$ correspond to the sales of the respective types of vehicles. This weighted EIS score is computed for all years with sales in both hybrid and non-hybrid vehicles, for each model of vehicle. For years where only non-hybrid 
cars were sold, a regular average was used to compute EIS. Using these new values for EIS, the S-curve for both hybrid and non-hybrid cars was constructed and is shown in Figure 4. From this curve, the lowest Energy Impact score for weighted Hybrid and Non-hybrid cars can achieve is 10, which saturates around year 2075. Practically, 95\% of the sustainability would be achieved around the year 2042 (EIS $=10.5$ ).

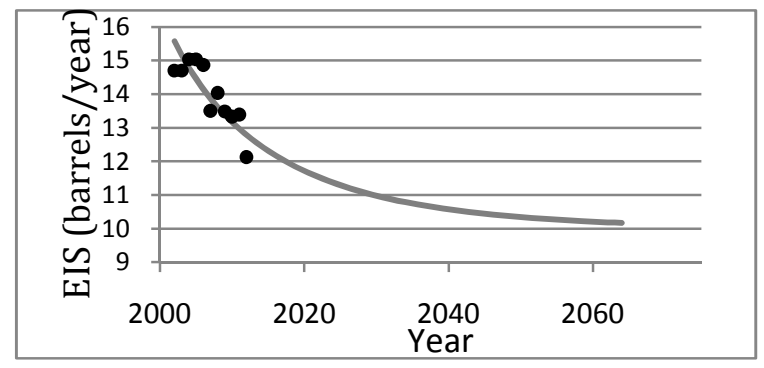

Fig. 4. Combined Hybrid and Non-Hybrids S-curve

\section{Discussion}

From our results, it was found that there was a significant relationship between total sales and the proportion of hybrid sales, model, and year variables. In particular, the higher the percentage of hybrid cars sold, the higher the overall sales for that model of vehicle. This result is important, because while the proportion of hybrid sales may not directly predict total sales, it indicates a positive trend between these two variables. In other words, a higher proportion of hybrid sales is associated with more success for a model of vehicle, encouraging car manufacturers to consider strategies for increasing hybrid car sales. This result could be attributed to the relationship between the proportion of hybrid cars produced and the success of a particular car manufacturer. From a policy-making and consumer standpoint, identifying factors that affect the overall sales of vehicles could prove useful for making decisions regarding the purchasing of vehicles.

From the game theory implementation, it was found that it would benefit all competing companies to have a higher percentage of hybrid sales. Since we found that the proportion of hybrid cars affects sales in the demand model, then we looked at different strategies that companies can make regarding the proportion of hybrid cars to produce and how it would affect their sales. For all three scenarios, the results suggest that the best strategy for all companies is to increase hybrid sales. These results show how hybrid technology is imperative for automobile companies to incorporate in order to be competitive. Further analysis could include a more in-depth analysis on the effect of brand positioning and company size on market shares.

The third step of the methodology involved technology forecasting of hybrid and non-hybrid engines. Using the sustainability metric, Energy Impact Score, we generated a total of $3 \mathrm{~S}$-curves corresponding to the EIS of the Toyota Camry hybrid, the Toyota Camry non-hybrid, and a combined hybrid and non-hybrid curve. It is important to note that the backwards S-curve shown in the model represents the tail-end of the S-curve because it already passed the inflection point for the years 
2002-2013. Our results showed that the saturation level of the EIS for hybrid vehicles is almost half of non-hybrid vehicles. This can be taken as justification as for why automotive makers should invest in a larger proportion of hybrid sales. That is, nonhybrid vehicles will likely never achieve the same level of sustainability as hybrid vehicles. In addition, since hybrid engines are predicted to continually improve in sustainability for the next 10 years, there is further motivation for automotive manufacturers to invest in hybrid vehicles.

\section{Conclusion}

This paper explored the factors that impact overall sales, and found that the proportion of hybrid sales, the year, and the type of car significantly influenced sales. It was also found that behavior of competitors' impacted the overall sales of a particular vehicle, highlighting the need for companies to consider the proportion of hybrid sales in competitive analysis. Lastly, we found justification for investment in hybrid technologies since improvement to sustainability scores are predicted to increase substantially for the next 10 years. Future work should incorporate larger databases of vehicle sales information in order to achieve a more generalizable model for predicting sales trends. In addition, we recommend that policies that encourage adoption of hybrid technologies (such as tax incentives) could benefit both automobile makers and consumers. Other measures of sustainability (such as amount of energy used to manufacture batteries) should be considered in future research regarding sustainability in order to increase the validity of the results.

\section{References}

[1] Lowery, E.: Energy Diversity as a Business Imperative, The Futurist

[2] Rius, B.: Electric Drive Design for Hybrid Electric Vehicle Optimum Fuel Efficiency. Shaker Verlag (2008)

[3] Schweinsberg, C.: U.S. Hybrid Sales Hit 2 Million Mark, Wards (2011)

[4] Khaligh, A.: Plug-in hybrid electric vehicles: sustainable driving to 21 st energy future. In: IECON 2010 (2010)

[5] Cook, H.E.: Decision-Based Design: Making Effective Decisions in Product and Systems Design, The Role of Demand Modeling in Product Planning

[6] Wassenaar, H.J., Chen, W., Cheng, J., Sudjianto, A.: Enhancing discrete choice demand modeling for decision-based design. In: Design Engineering Technical Conferences, Chicago, IL (2003)

[7] Myerson, R.B.: Game Theory: Analysis of Conflict. Harvard University Press (1991)

[8] Meyer, P.S.: A Premier on Logistic Growth and Substitutions: The Mathematics of the Loglet Lab Software. Technological Forecasting and Social Change 61(3), 247-271 (1999)

[9] Leyton-Brown, K., Yoav, S.: ssentials of Game Theory: A Concise, Multidisciplinary Introduction. Morgan and Claypool Publishers, San Rafael (2008)

[10] Cities, C.: Vehicle Buyer's Guide, Technical Report No. 